\title{
Aeolian sand transport in out-of-equilibrium regimes
}

H. Selmani ${ }^{1,4}$, A. Valance ${ }^{1}$, A. Ould El Moctar ${ }^{2}$, P. Dupont ${ }^{3}$, and R. Zegadi ${ }^{4}$

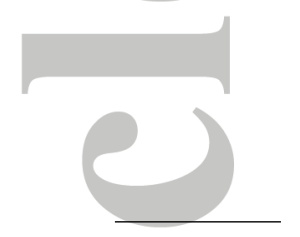

H. Selmani, selmani.h@univ-setif.dz

${ }^{1}$ Institut de Physique de Rennes, UMR

UR1-CNRS 6251, Université de Rennes 1,

35042 Rennes cedex, France.

${ }^{2}$ Laboratoire de Thermique et Energie de

Nantes, Polytech. Nantes, CNRS UMR

6607, 44306 Nantes, France.

LGCGM, INSA de Rennes, Campus

Beaulieu, 35043 Rennes, France.

${ }^{4}$ Laboratoire de Mécanique de Précision

Appliquée, Institut d'Optique et Mécanique

de précision, Université Ferhat Abbas Sétif

1, Algeria.

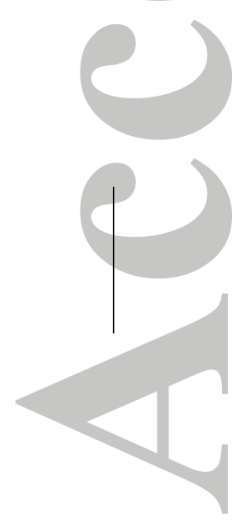


We investigate the relaxation process towards the equilibrium regime of saltation transport in the context of non-uniform conditions. Relaxation phenomena can be described in terms of a characteristic length scale which measures the distance for the particle flux to adapt to a spatial change in flow or boundary conditions. We conducted wind tunnel experiments to document the influence of the upwind mass flux on the relaxation process. For zero upwind mass flux conditions, the relaxation process is monotone and the relaxation length is independent of the wind strength. In contrast, for non-zero upwind flux conditions (obtained by releasing particles in the flow from a finite height), the relaxation process is non-monotone and is well captured by damped harmonic oscillations. Importantly, the relaxation length increases with increasing air flow velocity but is almost insensitive to the magnitude of the upwind flux. Our experimental outcomes clearly indicate that the relaxation of far from equilibrium transport regimes strongly deviates from a simple exponential behavior.

\section{Keypoints:}

- The saturation length is independent of the wind strength in the case of zero upwind mass flux conditions.

- In the case of finite upwind flux conditions (obtained by releasing particles in the flow from a finite height), the relaxation process is non-monotone and is well captured by a damped harmonic oscillation.

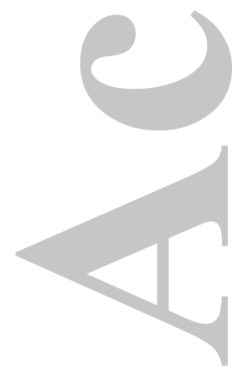


- The relaxation length determined in the latter case scales linearly with the friction velocity contrasting with the former case.

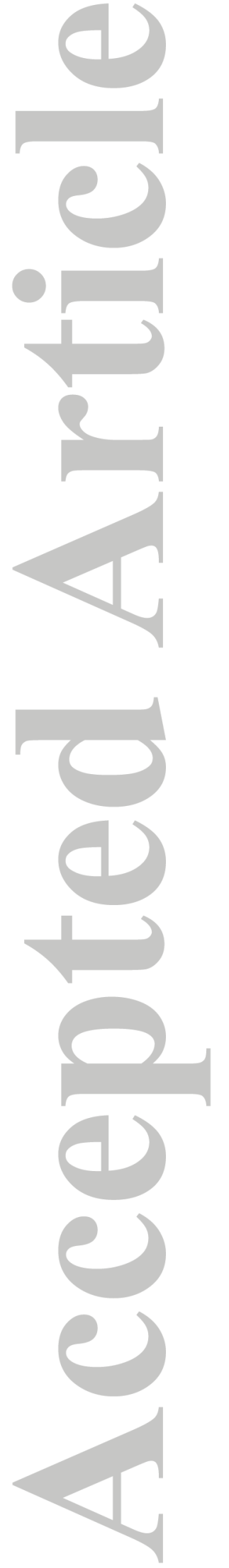




\section{Introduction}

Saltation is known as the main mode of sediment transport in air and characterizes the motion of the particles jumping along the sand surface in ballistic trajectories [Bagnold, 1941]. When a sufficiently strong wind blows over a sand bed, aeolian transport of sand is initiated and an equilibrium between air flow and transported particle is eventually achieved. This equilibrium state is characterized by an equilibrium or saturated mass flux which is an increasing function of the wind strength. Up to now, most researches were focused on the description of the equilibrium state of transport where erosion and deposition processes balance exactly [Bagnold, 1941; Shao, 2008; Durán et al., 2011; Kok et al., 2012]. A further interesting and important issue is the description of the out-ofequilibrium transport. If we consider a situation where we have a sudden increase or decrease in wind velocity, the mass flow rate does not adapt instantaneously to its new equilibrium value. This transient lasts a certain characteristic time (or occurs over a certain characteristic distance) for the new grains extracted from the bed to reach the air velocity and for the particle concentration to relax toward its new equilibrium value

[Durán et al., 2011; Valance et al., 2015]. During this transient process, the air speed within the transport layer has also to decrease to its equilibrium value because of the negative feedback of transport on the wind. Bagnold [1941] was the first to evidence that the saturation process takes a finite distance.

When brought slightly out of equilibrium, the particle laden air flow is shown to relax to its equilibrium state via an exponential behavior which can be modeled by a linear first order differential equation for the mass flux $Q$ [Andreotti et al., 2010; Pähtz et al.,

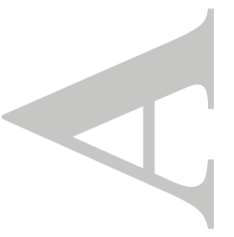


2013, 2015]:

$$
\frac{\partial Q}{\partial x}=-\frac{Q-Q_{s a t}}{L_{s a t}}
$$

where $L_{s a t}$ is the characteristic length scale of the relaxation process and $Q_{\text {sat }}$ is the saturated state corresponding to the equilibrium state. It is worth noting that the mass flux $Q$ is nothing but the product of the transported particle mass per unit area by the average particle velocity. Hence, the physics of the saturation process strongly depends on the interplay between these two physical variables. This equation describes a spatial inhomogeneous situation but an analog equation involving a characteristic time scale $\tau_{\text {sat }}$ can be written for unsteady transport. The solution of Eq. 1 simply reads:

$$
Q(x)=Q_{s a t}+\left(Q_{x_{0}}-Q_{s a t}\right) \exp \left(-\left(x-x_{0}\right) / L_{s a t}\right)
$$

where $Q_{x_{0}}$ is the upwind mass flux at the position $x=x_{0}$. This simple exponential behavior is expected to provide a good description of the relaxation process as long as the upwind mass flux is not too far from the equilibrium saturated flux $Q_{\text {sat }}$. Andreotti et al. [2010] confirmed via wind-tunnel experiments that the final stage of the relaxation (i.e., for $\left.Q>Q_{\text {sat }} / 4\right)$ towards the saturated state is well described by Eq. (2). The first stage of the process (i.e., for $0<Q<Q_{\text {sat }} / 4$ ) which corresponds to the initial growth of the particle flux is however not captured by Eq. (2). In the initial growth phase, the erosion process is prevailing and is driven both by aerodynamic and impact entrainment. In the following, the characteristic length associated with this initial phase will be referred to as the amplification length $L_{a m p}$.

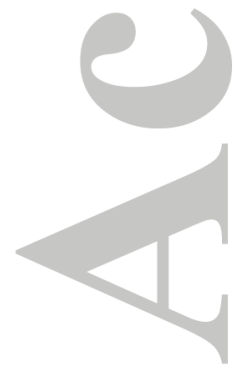


An alternative relaxation equation was proposed by Sauermann et al. [2001] to account for the whole process from the initial growth phase to the saturation one and reads:

$$
\frac{\partial Q}{\partial x}=-\frac{Q\left(Q / Q_{\text {sat }}-1\right)}{L_{\text {sat }}}
$$

This equation differs from Eq. (1) by the presence of a quadratic term in $Q$ which allows to describe the amplification stage for small $Q$. In contrast, for large particle flux $Q$ (i.e., close to $\left.Q_{\text {sat }}\right)$, we recover the simple linear relaxation law. This non-linear relaxation equation can be easily integrated and yields:

$$
Q(x)=\frac{Q_{0}}{Q_{0} / Q_{\text {sat }}+\left(1-Q_{0} / Q_{\text {sat }}\right) \exp \left(-x / L_{\text {sat }}\right)},
$$

with $Q_{0} \equiv Q(x=0)$.

Other experimental results indicate however more complex relaxation processes with an overshoot which is sometimes accompanied by an oscillation behavior. In particular, Bagnold [1941] reported wind-tunnel experiments showing that this type of complex relaxation occurs when the upwind mass flux $Q_{0}$ set at the entrance of the tunnel is set to a finite value. The latter situation with a finite upwind flux may appear a bit artificial at first sight but it is in fact intended to mimic a situation corresponding to a change in the basal boundary conditions, i.e., a transition from a rigid substrate to erodible bed with a finite upwind flux. This type of settings can be encountered in a large number of natural situations. The most prominent example is that corresponding to sand transport in a barchan dune field where the ground alternates rigid basal surfaces (i.e., inter-dune areas) and erodible sand beds (i.e., dune areas) [Ould Ahmedou et al., 2007].

In Bagnold experiments, the upwind flux is set with a hopper device applied to the tunnel roof. The Bagnold data can definitively not be described by a simple exponential

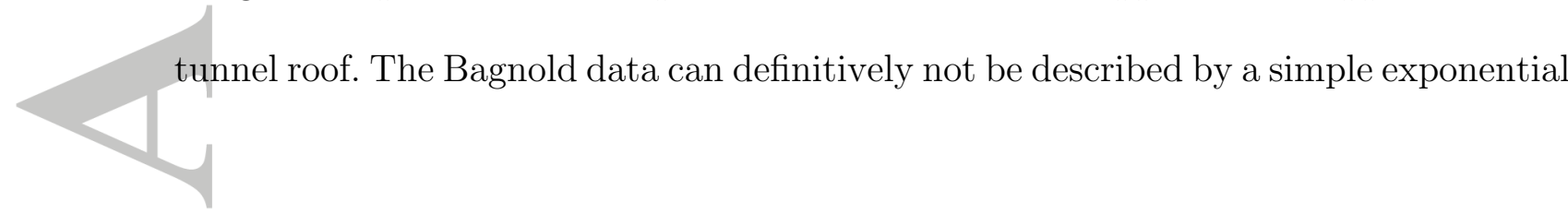


or quadratic non-linear relaxation but share instead some features of a damped harmonic oscillator (see Fig. 1). A formal relaxation equation for $Q$ based on a damped harmonic oscillator would read:

$$
\frac{\partial^{2} Q}{\partial x^{2}}+\frac{2}{L} \frac{\partial Q}{\partial x}+\left(\frac{2 \pi}{\lambda_{0}}\right)^{2}\left(Q-Q_{s a t}\right)=0
$$

where $L$ and $\lambda_{0}$ are two characteristic length scales. The latter stands for the natural wavelength oscillation of the undamped oscillator and the former is the length scale associated to the damping process. We can define a damping ratio $\zeta$ from these two length scales: $\zeta=\lambda_{0} / 2 \pi L$. For large damping ratio (i.e., $\zeta>1$ ), we get an exponential relaxation without oscillation:

$$
Q(x)=Q_{\text {sat }}+\left(Q_{0}-Q_{\text {sat }}\right)\left[\beta \exp \left(-x / L_{-}\right)+(1-\beta) \exp \left(-x / L_{+}\right)\right]
$$

with $L_{ \pm}=L /\left(1 \pm \sqrt{1-1 / \zeta^{2}}\right)$. The largest length scale $L_{-}$provides the characteristic distance for the saturation process while the smallest one $L_{+}$stands for the characteristic scale for the initial amplification process. In contrast, for small damping ratio (i.e., $\zeta<1$ ), we obtain damped oscillations with a wavelength $\lambda=\lambda_{0} / \sqrt{1-\zeta^{2}}$ obeying the following relation:

$$
Q(x)=Q_{s a t}+\left(Q_{0}-Q_{s a t}\right) \frac{\sin \left[(2 \pi / \lambda) x+\phi_{0}\right]}{\sin \phi_{0}} \exp (-x / L)
$$

where $L$ stands for the characteristic decay length of the oscillation. Expression (7) gives an excellent approximation to capture the experimental data of Bagnold (see Figure 1). Our conjecture that Eq. (5) provides a better framework to describe aeolian relaxation processes is supported by new wind-tunnel experiments reported in this letter.

Another important and still controversial issue concerns the physical mechanisms that govern the relaxation process. Sauermann et al. [2001] were the first to derive a theoretical 
expression for $L_{\text {sat }}$ based on the assumption that the relaxation length is governed by the efficiency of the erosion process due particle impacts (i.e., the splash process). Based on estimations of the elementary dune size in both subaqueous and aeolian environments, Hersen et al. [2002] argued that $L_{\text {sat }}$ is proportional to the drag length $L_{\text {drag }}=\left(\rho_{p} / \rho_{f}\right) d$, where $\rho_{p}$ and $\rho_{f}$ are respectively the particle and fluid density. Physically, the drag length corresponds to the distance needed for an inertial particular to equilibrate its velocity with that of the fluid. In an other point of view, Andreotti [2004] proposed that $L_{\text {sat }}$ is driven by the negative feedback of the transported particles on the wind to take place. This feedback mechanism is also governed by the drag length. Direct measurements of the saturation length through wind-tunnel experiments [Andreotti et al., 2010] confirmed that $L_{s a t}$ is on the order of magnitude of the drag length and independent of the wind strength as expected in the hypothesis that the relaxation length is closely linked to the drag length.

Pähtz and co-workers [Pähtz et al., 2013, 2014, 2015] recently proposed an expression of the saturation length based on the saltation length $L_{\text {salt }}$ and thus governed by the average speed of sand particles traveling above the sand bed: $L_{\text {sat }} \propto L_{\text {salt }} \approx V_{p}^{2} / g$. As the grain velocity in steady regime of saltation is invariant with the wind speed [Ho et al., 2011; Valance et al., 2015], the saltation length is expected to be independent on the wind strength like the drag length.

The present experimental study does not give a definite answer concerning the pertinent physical mechanisms of the relaxation process but provide clear evidences that the relaxation process toward the saturated regime of transport is strongly dependent on the

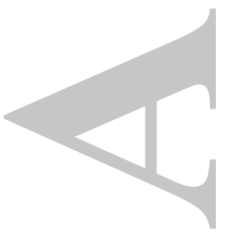


way how the system is brought out of equilibrium. We carried out extensive wind-tunnel experiments to characterize the spatial evolution of the sediment flux $Q(x)$ and relaxation towards the equilibrium state of transport with controlled upwind conditions in terms of mass flux $Q(x=0) \equiv Q_{0}$. We confirm the saturation length is invariant with the wind strength for zero upwind flux conditions and show that for finite upwind flux $\left(Q_{0}>0\right)$ obtained by releasing particles in the flow from a finite height, the saturation length is an increasing function of the wind strength contrasting with the results of the literature.

The paper is organized as follows. First, the wind tunnel facility and experimental protocols are presented in Section 2. Results and analyses are given in Section 3 while Section 4 provides conclusion and outlook.

\section{Experimental set-up and Protocol}

The experiments reported here were performed in a $6.6 \mathrm{~m}$ long wind tunnel with a cross-section of dimensions $0.27 m \times 0.27 m$ where the nominal air velocity $U_{\infty}$ (i.e., the air speed outside from the boundary layer) can be varied between 0 to $20 \mathrm{~m} / \mathrm{s}$ (see Ho et al. [2011, 2014] for further details). At the upwind edge of the tunnel, a hopper is installed on the roof of the wind-tunnel and delivers a constant and prescribed upwind sand flux $Q_{0}$ (see Fig. 2). The floor of the tunnel is covered with a sand layer whose stream-wise length $L_{\text {bed }}$ was varied from 0.2 to $6 \mathrm{~m}$. The upwind edge of the sand bed is located right below the feeding system (i.e., the hopper). We used natural sand with a median diameter $d=200 \mu \mathrm{m}$ and density $\rho_{p}=2650 \mathrm{~kg} / \mathrm{m}^{3}$. The air speed is measured by using a Pitot tube located at a distance of $6 \mathrm{~m}$ from the beginning of the tunnel and set at a height of $130 \mathrm{~mm}$

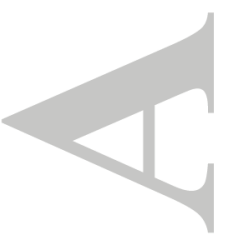


from the floor. The Pitot tube is outside from the boundary layer and is used to assess the nominal air velocity $U_{\infty}$.

We performed experiments with increasing bed length $L_{b e d}$ from 0.2 to $6 m$ (with an increment of $0.5 \mathrm{~m}$ ) for various air speeds (from 6 to $10 \mathrm{~m} / \mathrm{s}$ ) and three different upwind sand fluxes $Q_{0}\left(Q_{0}=0,35.6\right.$ and $\left.69.5 \mathrm{~g} / \mathrm{m} . \mathrm{s}\right)$. The non-zero values of upwind fluxes correspond to saturated values of saltation transport obtained at $U_{\infty}=7.5$ and $9.2 \mathrm{~m} / \mathrm{s}$, respectively.

Each run follows the same protocol. The sand bed is prepared with a uniform thickness of $2 \mathrm{~cm}$ and a given stream-wise bed length $L_{b e d}$. The sand bed surface is flattened before each run. The fan of the tunnel is tuned at a given wind speed while the hopper is opened at the same time delivering a prescribed upwind flux. A run lasts typically a few minutes (typically between 1 and 10) in order to collect a measurable amount of sand at the exit of the tunnel. After each run, the collected sand is weighed and the resulting outgoing flux $Q\left(x=L_{b e d}\right)$ is determined as follows: $Q=M /(T \times W)$, where $M$ is the mass of the collected sand, $T$ the duration of the run and $W$ the wind-tunnel width. This procedure allows to document the evolution of the mass flux $Q(x)$ as a function of the downstream distance $x$ (measured from the upwind edge of the sand bed) and to estimate the characteristic distance needed to reach the saturated state of transport starting from different out of equilibrium situations.

Lastly, it is important to make a few comments about the conditions in which the particles from the hopper are released in the tunnel in the case of non-zero upwind flux. The particles are released in the flow from the roof of the tunnel. Thus, they enter the flow with no horizontal momentum component but they are immediately dragged

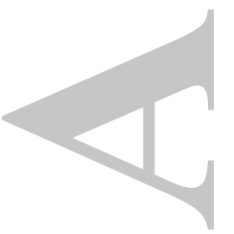


with the air flow. As a consequence, the particles do not fall straight down but follow a ballistic trajectory and impact the bed obliquely. Importantly, the fall time (which is about $\sqrt{2 h / g}=0.25 \mathrm{~s}$ where $h=0.27 \mathrm{~m}$ is the height of the tunnel) is larger than the characteristic time $\tau_{e q}$ for the particle to equilibrate its horizontal velocity with that of the wind $\left(\tau_{e q}=L_{d r a g} / U_{\infty}\right)$ which varies from 0.1 to $0.05 s$ for wind speed ranging from 5 to $10 \mathrm{~m} / \mathrm{s}$ respectively. As a result, the particles that are launched from the roof will therefore impact the bed with a horizontal velocity which is close to that of the wind. It is thus important to keep in mind that the upwind conditions correspond to a transport state with a finite flux where the mean particle speed is far in excess in comparison with that obtained in a steady and saturated state.

\section{Experimental Results}

From Bagnold observations (see Figure 1), the relaxation process is expected to be completely different according to the upwind flow conditions (i.e., the mass flux prescribed at the upwind edge of the wind tunnel). We first revisit the situation where the upwind flux is set to zero and then investigate the situation where there is a finite upwind flux.

\subsection{Zero upwind mass flux conditions}

Figure 3.a shows the spatial evolution of the mass flux $Q(x)$ for different wind speeds in the situation where the upwind mass flux $Q_{0}$ is set to zero. The mass flux increases monotonously with increasing distance until an asymptotic value corresponding to the saturated value is reached. As expected, the saturated value increases with increasing wind strength.

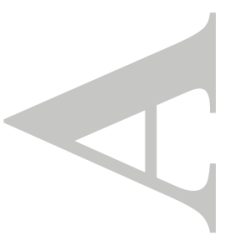


As previously emphasized by Andreotti et al. [2010], the relaxation process exhibits two different characteristic length scales, one characterizing the initial stage of mass flux amplification $\left(L_{a m p}\right)$ and the other corresponding to the exponential relaxation towards the saturated state $\left(L_{\text {sat }}\right)$. To extract the characteristic length scales, we employ three different methods. The first one is that proposed by Andreotti et al. [2010] where the amplification length is defined as the distance to reach $Q_{\text {sat }} / 4$ and the saturation length is determined by approaching the last stage of the relaxation process (i.e., when $Q>Q_{\text {sat }} / 4$ ) via a simple exponential relaxation (cf. Eq. 2). The second one relies on the solution of the non-linear relaxation equation (cf. Eq. 4). We uses the same criterion as previously to define the characteristic amplification length (i.e., $\left.Q\left(x=L_{a m p}\right)=Q_{s a t} / 4\right)$ which yields: $L_{a m p}=L_{s a t} \ln \left(Q_{0} / 3 Q_{\text {sat }}\right)$. The third method is based on the solution of an over-damped harmonic oscillator (cf. Eq. 6) where $L_{-}$provide the saturation length and $L_{+}$the amplification one.

The three methods yield similar results for the amplification and saturation length (see Table S2 in the supporting information and Fig. 3) except for the lowest wind speeds where the over-damped oscillator solution fails in describing properly the relaxation behavior. All three methods reveal that the amplification length $L_{a m p}$ decreases with increasing wind speed while the saturation length $L_{\text {sat }}$ does not show any significant variation with the air flow velocity. These finding confirm the experimental trends found by Andreotti et al. [2010]. Our measurements show that the magnitude of the saturation length is of order of $0.4 m$, which is about the same value as that obtained by Andreotti et al. [2010] but with sand grains twice smaller than ours $(0.120 \mathrm{~mm}$ against $0.200 \mathrm{~mm})$. Those direct

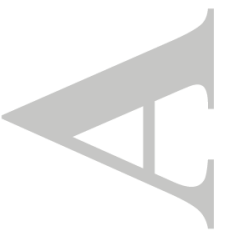


measurements of the saturation length do not indicate an increase of the latter with the grain size and thus contradict the indirect measurements of Andreotti et al. [2010] which show a linear scaling of the saturation length with the particle diameter. Further experimental investigations are thus needed to provide with a definite statement about the linear scaling of the saturation length with the particle diameter.

Finally, it is worthwhile to note that none of the new methods introduced here (i.e., nonlinear and over-damped relaxation) provides us with a more robust way to determine the amplification and relaxation lengths. The nonlinear relaxation solution is doing a bad job for describing the final stage of the relaxation process resulting in the underestimation of the saturation length by a factor two while the over-damped harmonic solution fails in capturing properly the relaxation process for the lowest wind speeds.

\subsection{Non-zero upwind mass flux conditions}

In the case of non-zero upwind mass flux, the relaxation process exhibits a contrasting behavior with a non-monotone evolution (see Fig. 4). The spatial evolution of the mass flux $Q(x)$ presents indeed a peak value at a finite distance that is greater than the incoming mass flux $Q_{0}$ and also greater than the saturated value. The overshoot is clearly caused by the process of impact entrainment triggered by the particles released upwind and appears in almost all situations, whether the value of upwind mass flux is higher or smaller than the saturated flux. As matter of fact, even if the falling height of the seeded particles is moderate, they are accelerated by gravity and dragged by the air flow to velocities larger than the particle velocity in the saturated state. Thus they should produce a greater number of ejecta when they collide with the bed [Beladjine et al., 2007] and this results

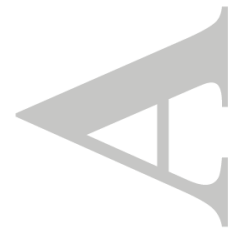


in an overshoot in the mass flux. Afterward, a decelerating process takes place due to the negative feedback of the particles on the wind. This situation contrasts with the case where the particles start from rest and are progressively accelerated by the air flow and strive to reach the minimum required velocity to saltate continuously.

The peak value $Q_{\max }$ is found to increase both increasing wind strength and upwind mass flux. A detailed analysis indicates that the latter obeys the simple following scaling: $\left(Q_{\max }-Q_{\text {sat }}\right) / Q_{0} \approx 0.4\left(u^{*} / u_{c}^{*}\right)$, where $u^{*}$ is the friction velocity characterizing the saturated state of transport and $u_{c}^{*}$ is the static threshold velocity. To estimate the friction velocity, we used the correlation between the nominal velocity $U_{\infty}$ and the friction velocity $u^{*}$ determined by Ho et al. [2011] in comparable conditions (similar sand grains and tunnel length): $u^{*}=0.13 \times U_{\infty}-0.32$.

In our experiments, the overshoot is attributed to the pre-existing upwind sand flux and disappears when the upwind flux vanishes. In the experimental work of Shao and Raupach [1992], an overshoot of the mass flux is also observed without any upwind mass flux. The source of this contrasting behavior remains unclear.

The non-monotone evolution resembles the solution of a damped harmonic oscillator. We thus employed Eq. 7 to extract the characteristic length scales of this behavior, that is the relaxation length $L$ and the wavelength $\lambda$ of the damped oscillation (see Fig. 5). First, both characteristic length scale $L$ and $\lambda$ are found to increase with the wind strength. Second, we do not see any significant effect of the magnitude of the upwind mass flux $Q_{0}$ on these lengths. These results suggest that the relaxation process is mostly driven by the wind speed and not by the intensity of the bombardment of the released particles.

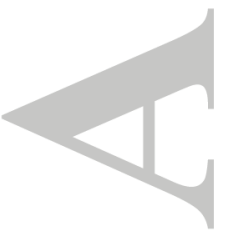


Although the uncertainty of the measurements, the relaxation length $L$ and the wavelength $\lambda$ seems to be compatible with a linear scaling law with the friction velocity (see the inserts of Fig. 5). This result contrasts with the invariance of the saturation length in the case of zero upwind mass flux and shows that the relaxation process is strongly dependent on the way we bring the system out from the equilibrium.

Additionally, we find that the position $L_{\text {peak }}$ of the peak value of the mass flux is also an increasing function of the wind strength and independent of the upwind mass flux. Importantly, the three length scales $L, \lambda$ and $L_{\text {peak }}$ are intimately correlated since we have: $\lambda \approx 4 L$ and $L_{\text {peak }} \approx L / 2$. The issue that remains to be further elucidated is to identify the physical mechanisms which control these length scales.

\section{Conclusion}

We carried out wind tunnel experiments to document the relaxation process towards the equilibrium regime of aeolian sand transport by varying the upwind mass flux. The main outcomes of this experimental study follow. (i) We first confirm the experimental outcomes of Andreotti et al. [2010], that is the saturation length is independent of the wind strength in the case of zero upwind mass flux conditions. (ii) Second, we show that in the case of finite upwind flux conditions (obtained by releasing particles in the flow from a finite height), the relaxation process is non-monotone as first evidenced by Bagnold [1941] and is well captured by a damped harmonic oscillation. (iii) Third, the relaxation length determined in the latter case scales linearly with the friction velocity contrasting with the former case.

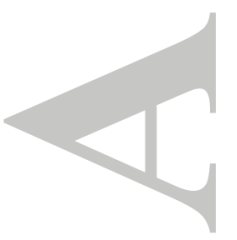


It is important to stress here that conditions with a pre-existing upwind sand flux can be encountered in a wide variety of natural situations. In desert environment, one can experience at least two fundamental different situations in terms of sand transport. The first one (probably the most studied) corresponds to transport on an erodible sand bed and it is encountered on sandy deserts where there is an inextinguishable source of sand. In this case, out-of-equilibrium transport is produced by a temporal or spatial variation of the wind strength. In contrast, the second situation corresponds to transport over a basal substrate whose nature exhibits spatial inhomogeneities (i.e., transition from a rigid bed to a particle bed). This situation is typical from desert areas with a low availability of sand like in barchan dune field areas.

Our experimental outcomes indicate that the relaxation of far from equilibrium regimes can not be described by a simple exponential behavior and requires the development of more sophisticated theoretical approaches in order to capture the complexity of far from equilibrium transport processes.

Finally, the oscillatory behavior of the relaxation process together with a characteristic length scale that increases with increasing wind speed observed in the case of a finite upwind mass flux may have some important implications concerning the dynamic of sand dunes. Indeed, it is admitted [Claudin and Andreotti, 2006; Parteli et al., 2007] that the saturation length actually controls the initial size of sand dunes emerging from a flat sand bed. In the picture where the saturation length is governed by the drag length [Andreotti et al., 2010] that is independent of the wind speed, the minimal size of a dune is therefore invariant with the wind strength. Our new outcomes suggest that in the context

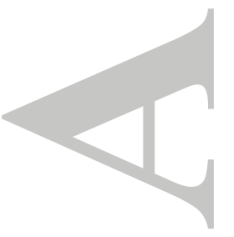


of barchan dunes lying on a rigid substrate and subject to a finite upwind mass flux, the minimal dune size could be dependent on the wind speed.

Acknowledgments. H.S. would like to thank the Algerian Ministry of Higher Education and Scientific Research for the financial support in the framework of program PNE 2015-2016. Data to support the figures in this manuscript are available in the support information.

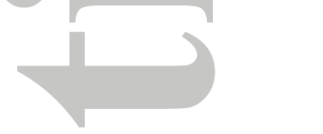

\section{5. *}

\section{References}

Andreotti, B. (2004), A two-species model of aeolian sand transport, Journal of Fluid Mechanics, 510, 47-70.

Andreotti, B., P. Claudin, and O. Pouliquen (2010), Measurements of the aeolian sand transport saturation length, Geomorphology, 123, 343-348.

Bagnold, R. A. (1941), The physics of blown sand and desert dunes, Methuen, New York. Beladjine, D., M. Ammi, A. Valance, and L. Oger (2007), Collision process between an incident bead and a three-dimensional granular packing, Physical Review E., 75, 061,305 .

Claudin, P., and B. Andreotti (2006), A scaling law for aeolian dunes on mars, venus, earth, and for subaqueous ripples, Earth and Planetary Science Letters, 252, 30-44.

Durán, O., P. Claudin, and B. Andreotti (2011), On aeolian transport: Grain-scale interactions, dynamical mechanism and scaling laws, Aoelian Research, 3, 243-270.

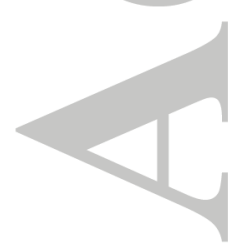


Hersen, P., S. Douady, and B. Andreotti (2002), Relevant length scale of barchan dunes, Physical Review Letters, 89, 264-301.

Ho, T, D., A. Valance, P. Dupont, and A. Ould El Moctar (2011), Scaling laws in aeolian sand transport, Physical Review Letters, 106, 094,501.

Ho, T. D., A. Valance, P. Dupont, and A. Ould El Moctar (2014), Aeolian sand transport: Height and length distribution of saltation trajectories, Aeolian Research, 12, 65-74.

Kok, J. F., E. Partel, T. Michael, and D. Bou Karam (2012), The physics of wind-blown sand and dust, Report on progress in Physics, 75, 106,901.

Ould Ahmedou, D., A. Ould Mahfoudh, P. Dupont, A. Ould El Moctar, A. Valance, and K. R. Rasmussen (2007), Barchan dune mobility in mauritania related to dune and interdune sand fluxes, Journal of Geophysical Research: Earth Surface, 112, F02,016.

Pähtz, T., J. F. Kok, E. J. R. Parteli, and H. J. Herrmann (2013), Flux saturation length of sediment transport, Physical Review Letters, 111, 218,002.

Pähtz, T., E. J. R. Parteli, J. F. Kok, and H. J. Herrmann (2014), Analytical model for flux saturation in sediment transport, Physical Review E, 89, 052,213.

Pähtz, T., A. Omeradžić, M. V. Carneiro, N. A. M. Araújo, and H. J. Herrmann (2015), Discrete element method simulations of the saturation of aeolian sand transport, Geophysical Research Letters, 42, 2063-2070.

Parteli, E. J. R., O. Durán, and H. J. Hermann (2007), Minimal size of a barchan dune, Physical Review E, 75, 011,301.

Sauermann, G., K. Kroy, and H. J. Herrmann (2001), Continuum saltation model for sand dunes, Physical Review E., 64, 031,305.

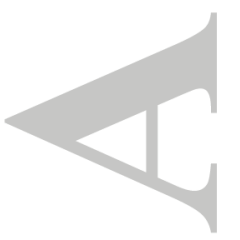


Shao, Y., and M. R. Raupach (1992), The overshoot and equilibration of saltation, Journal of Geophysical Research, 97, 559-564.

Shao, Y. (2008), Physics and modelling of wind erosion., Kluwer Academy, Dordrecht, Amsterdam.

Valance, A., K. R. Rasmussen, A. Ould El Moctar, and P. Dupont (2015), The physics of aeolian sand transport, Comptes Rendus Physique, 16, 105-117.

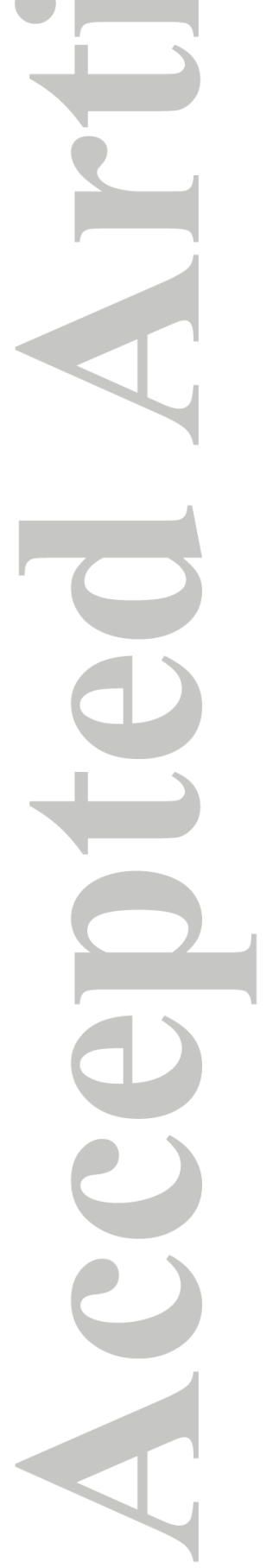




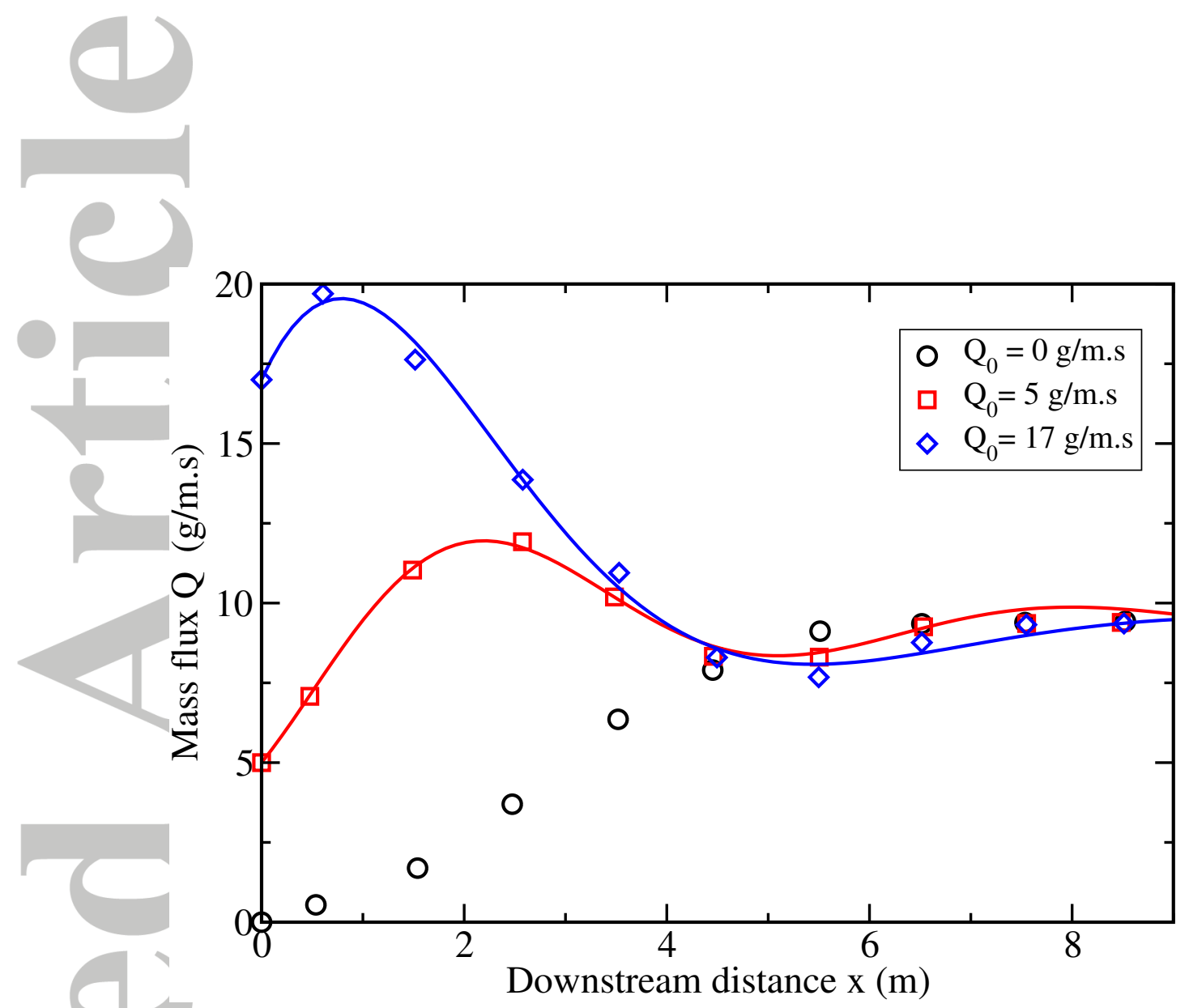

Figure 1. Variation of the mass flux along the downwind distance $x$ : Data from Bagnold [1941] for three different upwind flux conditions $Q_{0}=0,5$ and $17 \mathrm{~g} / \mathrm{m} . \mathrm{s}$ and a given nominal air velocity $U_{\infty}=4.9 \mathrm{~m} / \mathrm{s}$. The solid lines represent the best fit using Eq. (7). The parameters of the fit are given in Table S1 of the supporting information. 


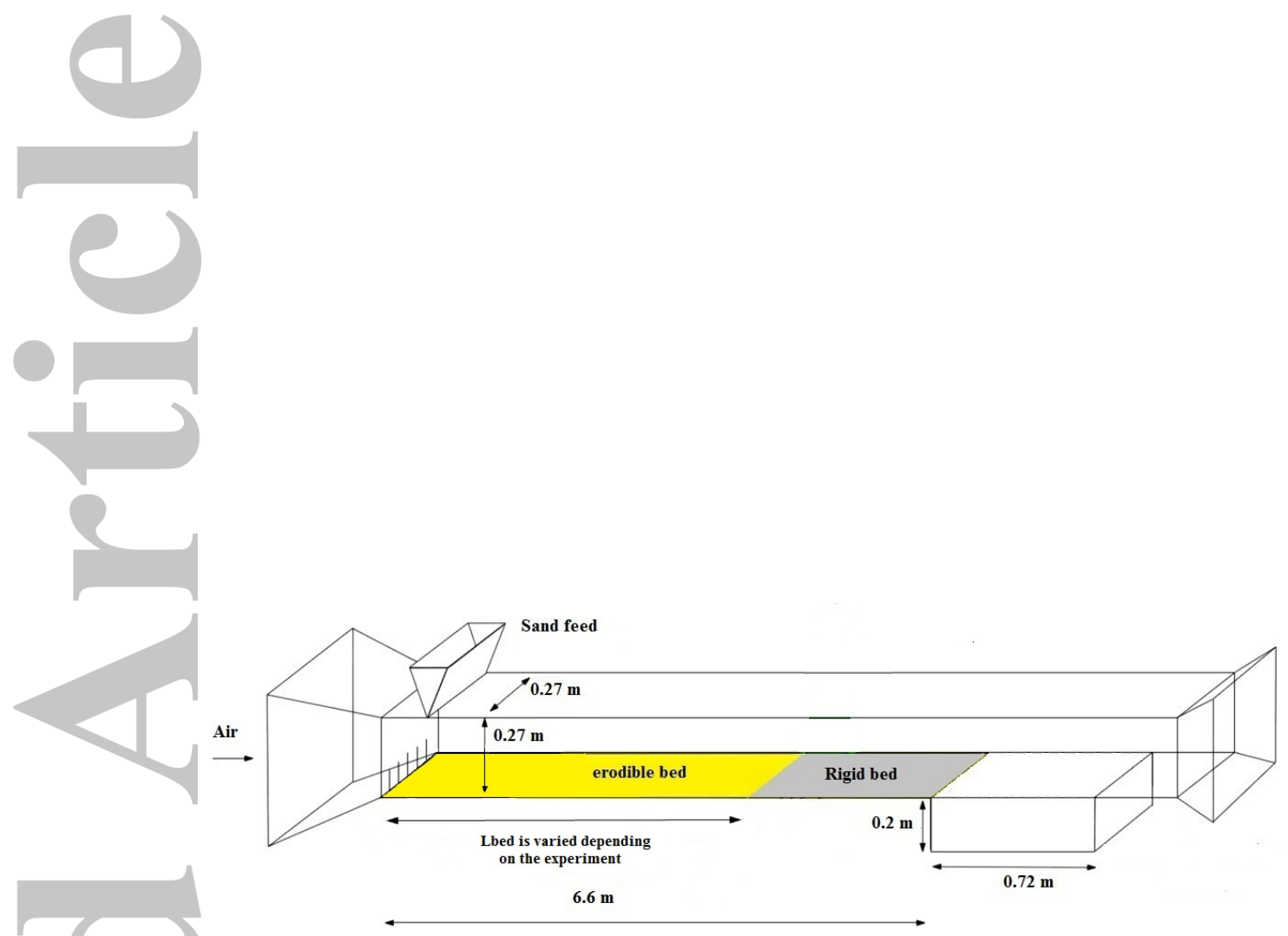

Figure 2. Schematic view of the wind tunnel

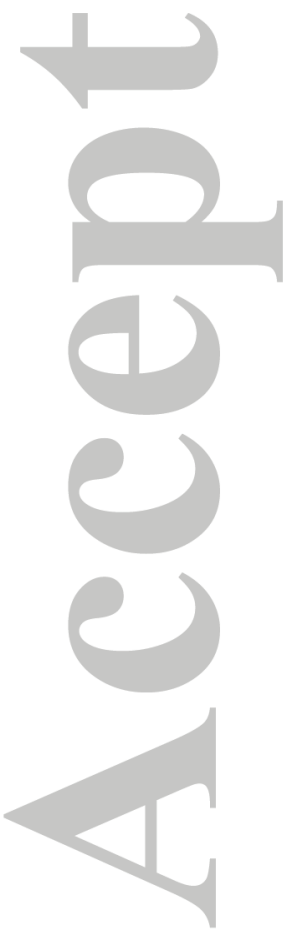




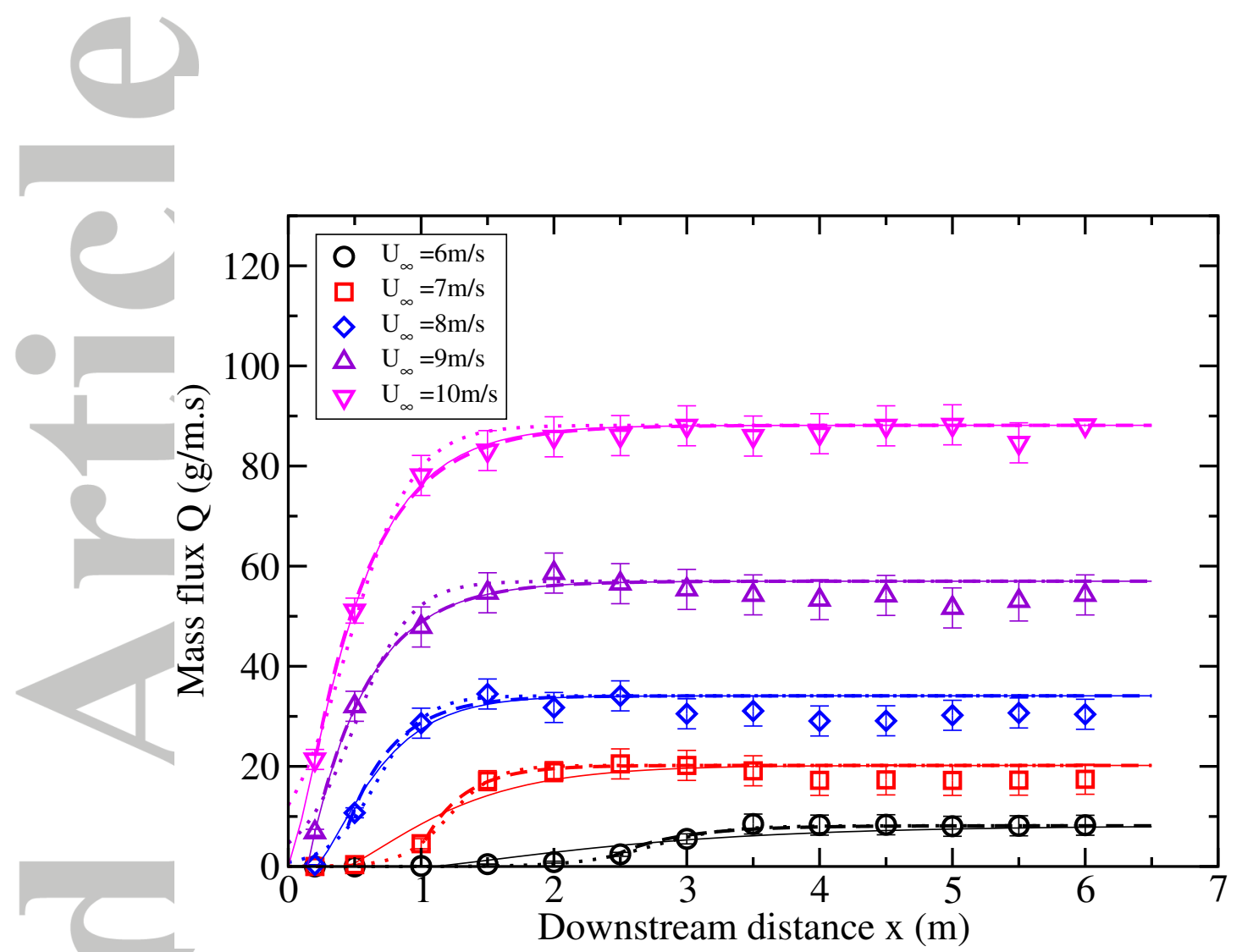

Figure 3. (a): Spatial evolution of the mass flux $Q$ along the downstream distance $x$ in the case of zero upwind flux (i.e., $\left.Q_{0}=0\right)$ for various air flow speeds $\left(U_{\infty}=6,7,8,9,10 \mathrm{~m} / \mathrm{s}\right)$. The dashed, dotted and solid lines represent respectively the best fit using Eq. (2), Eq (4) and Eq. (6) (see Table S2 in the supporting information for the values of the parameters). (b) and (c): Saturation and amplification length versus air flow speed obtained respectively from Eq. (2), Eq. (4) and Eq. (6). 


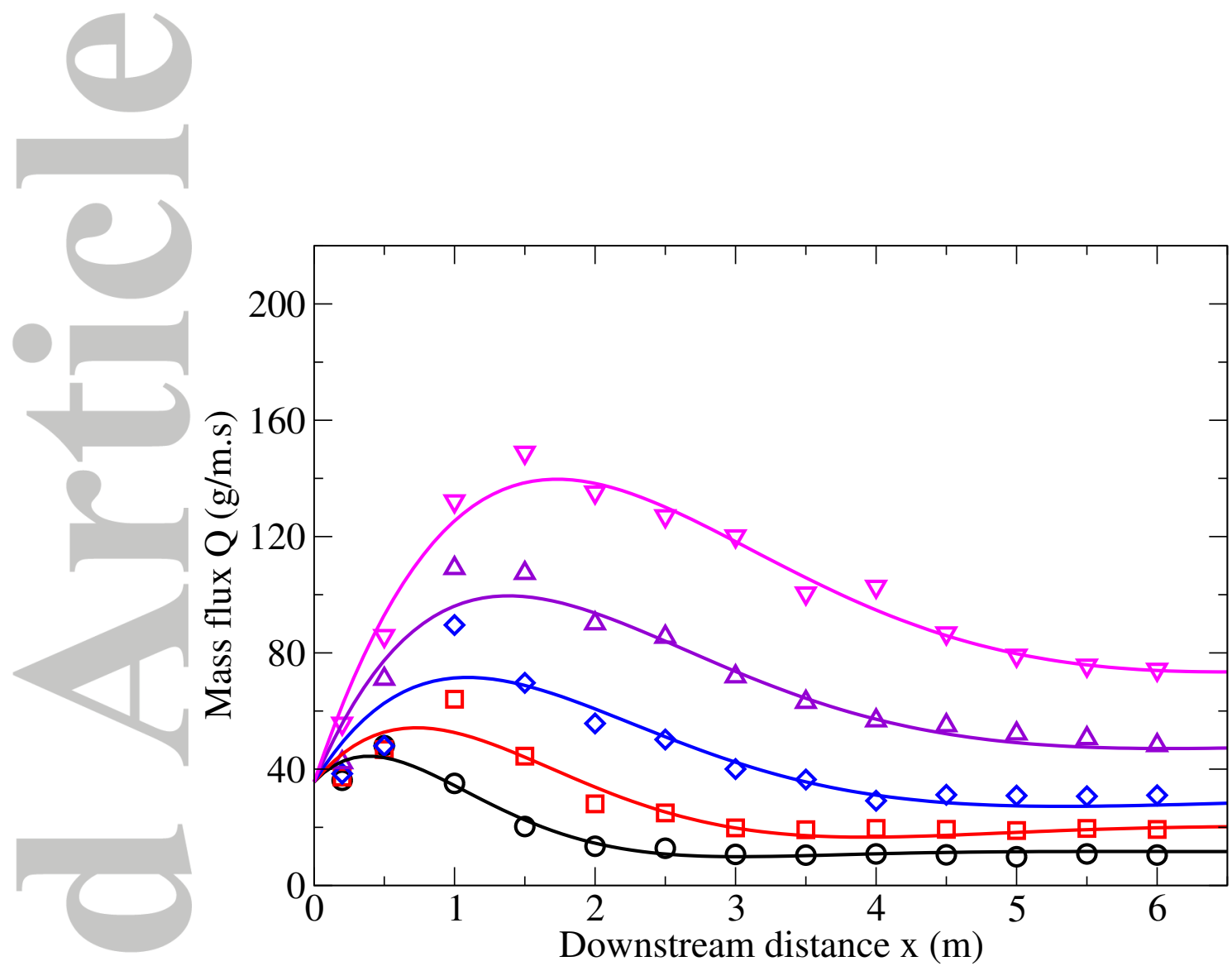

Figure 4. Spatial evolution of the mass flux $Q$ along the downstream distance $x$ in the case of non-zero upwind flux for various air flow speeds: (a) $Q_{0}=35.6 \mathrm{~g} / \mathrm{m} . \mathrm{s}$ and (b) $Q_{0}=69.5 \mathrm{~g} / \mathrm{m} . \mathrm{s}$; (॰) $U_{\infty}=6 \mathrm{~m} / \mathrm{s},(\square) U_{\infty}=7 \mathrm{~m} / \mathrm{s},(\diamond) U_{\infty}=8 \mathrm{~m} / \mathrm{s},(\Delta) U_{\infty}=9 \mathrm{~m} / \mathrm{s}$, and $(\nabla) U_{\infty}=10 \mathrm{~m} / \mathrm{s}$; The solid lines represent the best fit using Eq. (7) (see Table S3 in the supporting information for the values of the fitted parameters). 


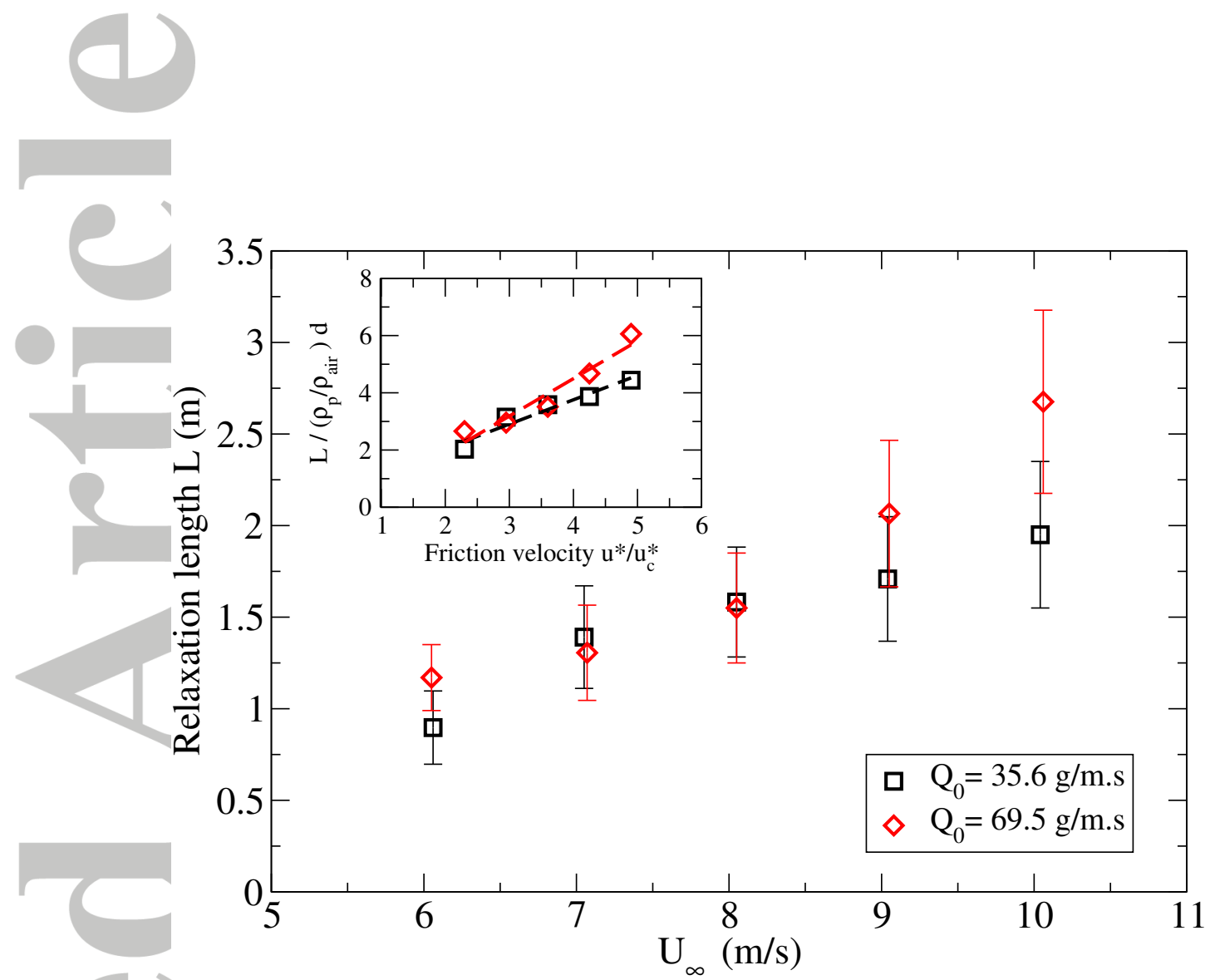

Figure 5. (a) Relaxation length $L$ versus the wind speed for two different upwind mass flux $Q_{0}=35.6$ and $69.5 \mathrm{~g} / \mathrm{m.s}$ (Insert: Relaxation length normalized by the drag length versus the friction velocity $u^{*}$ ). (b) Wavelength $\lambda$ and (c) peak position $L_{\text {peak }}$ versus the wind speed for the two same upwind mass flux. (Insert: Lengths normalized by the drag length versus the friction velocity $\left.u^{*}\right)$. 